\title{
PERSPECTIVES SUR LE DROIT QUÉBÉCOIS DE LA FAMILLE
}

Par Benoît Moore*

Resumé: O artigo apresenta um amplo panorama do direito de família no Québec-Canadá, a partir da entrada em vigor do Código Civil do Québec e de suas sucessivas reformas para atender novas demandas sociais.

Mots-clés: Droit de la famille - Canada; droit de la famille - Québec;

Le droit de la famille, terreau par excellence où se réalisent les valeurs d'une societé, a de tout temps été sujet à des changements souvent brusques et significatifs. Le droit québécois des trente dernières années illustre, à l'instar des autres pays occidentaux, ce phénomène. Une multitude de phénomènes sociaux, depuis les années 70 , tels les revendications dégalité entre les sexes, la perte d'influence de la religion, la croissance du phénomène de l'union de fait et des naissances hors-mariage, ont tous convergé pour imposer des réformes profondes et répétitives au corpus du droit de la famille. De ce fait, l'arrivée du Code civil du Québec, en 1994, ne fût ni l'occasion de la réforme de cette partie du droit privé, ni la fin de celle-ci.

* Professeur à la Faculte de Droit de l'Université de Montréal et avocat au Barreau du Québec.
En effet le droit québécois de la famille connaît depuis la fin des années 60 des changements sucessifs importants visant à relayer dans ses règles ces changements sociaux. Si un grand nombre de réformes a eu lieu, trois lignes directrices se dégagent de celles-ci. Tout d'abord, dans le contexte des discussions portant sur la réforme du Code civil, il avait été décidé de procéder à la réforme par étapes distinctives, livre par livre, et de débuter par celui de la famille. C'est ainsi que le Code civil du Québec en matière familiae a été adopté dès $1980 .^{1}$

\footnotetext{
${ }^{1}$ Loi institutant um nouveau Code civil et portant reforme du droit de la famille, L.Q. 1980, c. 39 entrée em vigueur pour la partie le 2 avril 1981 et pour partie le $1^{\text {er }}$ décembre 1981. À partir de cette époque et jusqu'à la mise em vigueur de l'ensemble de Code civil du Québec en 1994, le Québec avait deux Codes civils: le Code civil du Québec pour le livre de la famille et le Code civil du Bas-Canada pour le reste.
} 
Cette réforme législative, majeure, était essentiellement axée sur le principe de l'égalité juridique des membres de la famille, que ce soient les conjoints, les parents ou les enfants. Cette première réforme est suivie, en 1989, d'une intervention visant cette fois à assurer une certaine égalité économique entre les époux dans un contexte où de plus en plus d'union connaissent une rupture. ${ }^{2}$ Enfin, en juin 2002, s'inscrivant dans un courant occidental, législateur québécois est intervenu afin de créer un nouveau statut civil et établir des règles de filiation destinés tout particulièrement aux couples de même sexe, favorisant ainsi une recherche d'égalité entre les différents types d'union et de famille. ${ }^{3}$ C'est donc un processus continu de réforme que caractérise l'évolution du droit de la famille québécois. Nous verrons lé résultat de ces réformes multiples en présentant sommairement l'état du droit concernant l'union (I) puis la filiation (II).

\section{LA FAMILLE PAR L'UNION}

\section{A. Formation de l'union}

Le marriage. Le Québec faisant partie d'un État fédéral, il est nécessaire de signaler qu'il n'a pas en matière de famille, l'exclusivité de la compétence législative. La Loi constitutionnelle de $1867^{4}$ prévoit à

${ }^{2}$ Loi modifiant dle Code civil du Québec et d'autres dispositions législatives afin de favoriser l'égalité économique dês époux, L.Q. 1989, c. 55.

${ }^{3}$ Loi instituant l'union civile et établissant de nouvelles règles de filiation, L.Q. 2002, c. 6. La loi, pour l'essentiel, est em vigueur depuis le 24 juin 2002.

4 Loi iconstitutionnelle de 1867,30 \& 31 Victoria, ch. 3 (R.-U.). son article 91 (26) que le Parlement fédéral a la compétence relative au mariage et au divorce, alors que l'article 92 (12) donne aux provinces le contrôle de la célébration du mariage. Ce partage, effectué pour des raisons historico-religieuses, a toujours donné lieu à une interprétation restrictive de la compétence fédérale qui constitue une exception au principle selon lequel le droit privé relève de la compétence des provinces. Ainsi les tribunaux ont reconnu la compétence sur les conditions de fond du mariage au Parlement fédéral laissant la réglementation des conditions de forme aux législatures provinciales.

Pour le Québec la loi fédérale exige, pour la formation d'un mariage valide, l'existence d'un consentement libre et éclairé d'un homme et d'une femme. ${ }^{5}$ Le mariage est donc pour l'instant du moins, ouvert uniquement aux couples hétérosexuels. Cette limitation est par allieurs actuellement sous étude par une commission parlementaire qi a été formée suite à deux jugements ayant déclaré celleci discriminatoire en vertu de la Charte canadienne. ${ }^{6}$ Il est donc à prévoir que des développements sur ce sujet surviendront bientôt, soit par le biais des tribunaux, soit par le biais du législateur fédéral. Concernant les autres conditions de fond signalons que les époux doivent être âgés d'au moins 16 ans $^{7}$ et être libres tout mariage antérieur non

${ }^{5}$ Loi d'harmonisation no $1 \mathrm{du}$ droit fédéral avec le droit civil, L.C. 2000, c. 4, art. 5 .

${ }^{6}$ En Ontário: Halpern c. Canadá (A. G.), (2002) O.J. 2714 (Quicklaw). Au Québec: Hendricks c. Québec (Procureur general), [2002] R.J.Q. 2506 (C.S.). Ces deux décision sont en appel.

${ }^{7}$ Loi d'harmonisation no 1 du droit federal avec le droit civil, précitée, note 5, art. 6. 
dissous. ${ }^{8}$ Quant au empêchements familiaux ceux-ci sont minimaux, le mariage n'étant interdit qu'entre parents en line directe et, en ligne collatéralle, qu'entre frère/soeur, demi-frère/demie-soeur. ${ }^{9}$

Quant aux règles de forme, le mariage au Québec était, jusqu'en 1968, nécessairement religieux. Depuis cette époque ${ }^{10}$ il est posible de contracter un mariage exclusivement civil, mais ce type d'union ne représentait en 1999 que $29 \%$ des mariages. ${ }^{11}$ Il est en effet plus usité au Quebéc de contracter un mariage religieux, puisque la cérémonie religieuse vaut également comme mariage civil si elle respecte les conditions de la loi. Pour cela il est nécessaire que la société religieuse soit autorisée par le Ministre responsable de l'état civil à célebrer les mariages civils, ce qui est le cas pour l'ensemble des grandes religions et pour un bon nombre de regroupements religieux plus marginaux. ${ }^{12}$ La faible popularité du mariage civil s'explique entre autre par le fait que celui-ci devait être célebré au Palais de justice para un greffier de la Cour supérieure. Afin de rendre la célébration plus attrayante, il est maintenant possible, depuis l'été dernier, de se marier civilement devant un notaire ou certains officiers municipaux

\footnotetext{
${ }^{8}$ Loi d'harmonisation no $1 \mathrm{du}$ droit federal avec le droit civil, précitée, note 5, art. 7.

${ }^{9}$ Loi sur le mariage (degrés prohibés), L.R.C., c. $M-2.1$, art. 2 . c. 82 .

${ }^{10}$ Loi concernant le mariage civil, S.Q., 1968,
}

11 COMISSION DU DROIT DU CANADA, Au delà de la conjugalité, Ottawa, 2001, p. 148.

12 Art. 366 C.c.Q. [ce qui est généralement le cas]. dans tout lieu «respectant le caractère solennel de la cérémonie». ${ }^{13}$

L'union civile. Ce type d'union a été crée à l'été 2002 par le législateur québécois en réponse aux revendications de la communauté homosexuelle. La province n'ayant pas le pouvoir d'étendre le mariage aux personnes de même sexe - cette question étant de compétence fédérale - le legislateur québécois a décidé d'instaurer un nouveau type d'union en tout point identique au mariage. Conséquentemment à cette identité recherchée, les conditions de formation de cette union sont essentiellement les mêmes à l'exception, il va sans dire, qu'elle peut être contractée non seulement par un couple hétérosexuel mais également par un couple homosexuel. ${ }^{14}$ Tout comme le mariage, l'union civile peut être célébrée par un ministre du culte que y consent ${ }^{15}$ ou par un officier civil - incluant les officiers municipaux et les notaires - dans un lieu public et propice. Selon les premières statistiques, moins de deux cents couples auraient opté pour ce type d'union aprés six mois de d'application de la loi.

L'union de fait. Si ce type d'union état marginal, voire clandestin, il n'y a encore

${ }^{13}$ Art. 366 C.c.Q. et Régles sur la célébration du mariage civil ou de l'union civile, A.M. 2152, Gazette oficielle, partie 2, 12 mars 2003, p. 1506.

${ }^{14}$ L'union civile se retrouve dans le Code civil entre lãs dispositions portant sur le mariage et celles portant sur la filiation (art. 521.1 à 521.19 C.c.Q.). L'âge minimal requis est de 18 ans plutôt que 16 pour le mariage (art. 521.1 C.c.Q.).

15 Art. 521.2 C.c.Q. 
pas sis longtemps, il est en progression constante depuis les trente dernières annés et représentait au Québec, en 1999, 20\% des couples. ${ }^{16}$ Même si, nous le verrons, l'union de fait ne produit que fort peu d'effets civils, ce type de rapport est maintenant défini à l'article 61.1 de la Loi d'interpretation ${ }^{17}$ qui fonde l'union de fait sur l'existence d'une vie commune entre deux personnes (quelque soit le sexe) qui se présentent publiquement comme un couple. En cas d'incertitude la cohabitation depuis un an ou la cohabitation - sans durée minimale - et la présence d'un enfant commun, suffit.

\section{B. Effets de l'union}

Le marriage. Le marriage emporte l'application d'un régime primaire qui, aux termes de l'article 391 C.c.Q., est impératif et s'applique quelque soit le régime matrimonial choisi par les époux. Ce régime primaire assure tout particulièrement l'égalité entre les partenaires, égalité qui est la valeur dominante du droit de la famille québécois inscrite, depuis 1975, dans le Charte des droits et libertés de la personne du Québec qui, à son article 47 précise que les conjoints en mariage ont les mêmes droits et obligations. ${ }^{18}$ Cette disposition est aussi reprise dans le Code civil du Québec comme toute première disposition du régime

16 GOUVERNEMENT DU QUÉBEC, Um portrait statistique des familles et des enfants au Québec, Québec, 1999, p. 7.

17 L.R.Q., c. 1-16. Plusieurs lois définissent la notion de conjoint de fait. La définition de la loi d'interprétation ne s'applique que de façon subsidiaire.

18 Charte des droits et libertés de la personne, L.R.Q., c. C-12. primaire impératif. ${ }^{19}$ Conséquentement à ce principle le code impose que les conjoints contribuent aux chages du mariage en proportion de leurs facultés, ${ }^{20}$ précise que les époux assurent ensenble la direction morale et matérielle de la famille ${ }^{21}$ et choisissent de concert la résidence familiale. ${ }^{22}$ Chacun des époux conserve en mariage son nom, mettant ainsi de côté la coutume qui voulait que l'épouse prenne le non de son mari. ${ }^{23}$ La recherche de l'égalité entre les époux qui s'était amorcée en 1964 par une loi reconnaissant à la femme mariée, ${ }^{24}$ qui était à cet époque sous l'autorité maritale, la pleine capacité juridique. Afin de rendre cette égalité praticable les époux s'engagent solidairement pour les besoins courants de la famille ${ }^{25}$ et sont, lorsque nécessaire, présumés se représenter dans l'accomplissement des actes relatifs à la direction de la famille. ${ }^{26}$ Enfin, en cas de conflit, c'est le juge qui doit arbitrer les questions litigieuses. ${ }^{27}$

Le régime primaire prévoit également la protection de la résidence familiale ainsi que des meubles qui s'y trouvent et qui servent à l'usage de la famille. ${ }^{28}$ Ces biens ne peuvent

${ }^{19}$ Art. 392 C.c.Q.

${ }^{20}$ Art. 396 C.c.Q.

21 Art. 394 C.c.Q.

22 Art. 395 C.c.Q.

23 Art. 393 C.c.Q.

${ }^{24}$ Loi sur la capacite juridique de la femme mariée, L.Q. 1964, c. 66.

25 Art. 397, C.c.Q.

${ }^{26}$ Art. 398, C.c.Q.

27 Art. 400, C.c.Q.

28 Art. 401, C.c.Q. 
pas, en principe, être aliénés sans le consentement des deux conjoints et ce quelque soit l'identité du propriétaire. Ces mesures visent à protéger le lieu de vie des enfants et du conjoint non propriétaire et à assurer que les décisions majeures, dont celle du choix de la résidence, soient prises conjointement.

Sur le plan économique certains effets du régime primaire prennent racien lors de la dissolution de l'union. Il est possible pour un époux de demander une prestation compensatoire visant à l'indemniser pour l'enrichissement du patrimoine de son conjoint dont il aurait été la source tel qu'il en est, par example, de celui qui participe, sans rémunération, à l'entreprise de son conjoint. ${ }^{29}$ Ce recours, transcription dans le mariage de l'enrichissement injustifié, fut rendu nécessaire suite au refus par les tribunaux d'accueillir l'action de in rem verso entre époux au motif qu'il y aurait dans le régime matrimonial - même de la séparation de biens - une justification à l'enrichissement.

En 1989 le législateur pousse un cran plus loin la protection économique et crée le patrimoine familial qui est depuis l'incarnation par excellence de la conception associationiste qu'impose le législateur au mariage. Cette institution s'applique à tous les couples mariés depuis le $1^{\text {er }}$ juillet 1989 sans possibilité pour eux d'y déroger. ${ }^{30}$ Aux

\footnotetext{
29 Art. 427, C.c.Q.

${ }^{30}$ Loi modifiant le Code civil du Québec et d'autres dispositions législatives afin de favoriser l'égalité économique des époux, L.Q. 1989, c. 55. Cette loi s'applique également aux personnes mariées antérieurement au $1^{\mathrm{er}}$ juillet 1989 sous réserve pour
}

termes de ces dispositions il y a partage égal, au moment de la dissolution du mariage, de la valeur des biens inclus dans le patrimoine familial. Ceux-ci sont, aux termes de la liste limitative de l'article 415 C.c.Q.: 1) les résidences de la famille ou les droits qui en confèrent l'usage; 2) les meubles qui les garnissent et les ornent et qui servent à l'usage du ménage; 3) les véhicules automobiles utilisées pour les déplacements de la famille; 4) les droits accumulés durant le mariage au titre d'un régime de retraite. Ces biens sont inclus dans le patrimoine familial même s'ils ont été acquis avant le mariage. Par ailleurs sont exclus les biens reçus par l'un des époux en guise de sucession ou de donation. Le patrimoine familial ne donne aucun droit réel au conjoint non-propriétaire des biens, il ne fait qu'octroyer un droit de créance éventuel. ${ }^{31}$

Ce n'est qu'après l'application des règles impératives du régime primaire - dont le patrimoine familial - que s'appliquent celles du «régime secondaire» c'est-à-dire le régime matrimonial librement choisi par les conjoints dans un contrat de mariage notarié et conclu avant la célebration de l'union. ${ }^{32}$ En l'absence de choix par ceux-ci c'est,

eux de s'être exclux de l'application de la loi par acte notarié avant le 31 décembre 1990 (art. 42 de la loi).

31 Droit de la famille - 977, [1991] R.J.Q. 904 (C.A.). Ce droit est, en principe, équivalant à la moitié de la valeur réelle des biens inclus au moment du partage, ou encore pendant le mariage avec des fonds reçus para donation ou succession ou en remploi d'une telle somme ou d'un bien du patrimoine familial possédé avant le mariage. Voir articles 417 et 418 C.c.Q.

32 Art. 431 C.c.Q. 
depuis $1969,{ }^{33}$ le régime de la societé d'acquêts qui régit les conjoints. Ce régime allie l'independance de gestion que procure la séparation de biens et la protection économique de la communauté de biens. En effet, les conjoints durant l'union administrent, en principe, seuls leurs biens et lors de la dissolution du régime il y a partage en valeur des acquêts. ${ }^{34}$

Enfin l'article 585 C.c.Q. prévoit une obligation alimentaire entre les conjoints, cette obligation découlant de l'obligation de secours et d'assistance que se doivent les époux. ${ }^{35}$ Ce n'est par ailleurs que lors de la dissolution de l'union que prend racine dans le réel l'obligation alimentaire celle-ci puvant survivre au décès du débiteur ${ }^{36}$ ou au divorce. ${ }^{37}$ Des aliments peuvent également être octroyés lors d'une séparation de $\operatorname{corps}^{38}$ ou d'une nulitté e mariage. ${ }^{39}$

\section{L'union civile. Si le législateur} québécois a crée l'institution de l'union civile ce n'est qu'à défaut de ne pas avoir pu permettre le mariage netre conjoints de même sexe. L'ojectif avoué était donc d'établir une parfait entre les deux institutions. Pour ce

${ }^{33}$ Loi concernant les regimes matrimoniaux, L.Q. 1969 , c. 77.

${ }^{34}$ Les acquêts et les biens propres sont prévus aux articles 448 et suiv. C.c.Q. L'article 461 C.c.Q. prévoit que «[c]haque époux a l'administration, la jouissance et la libre disposition de ses biens propres et de ses acquêts».

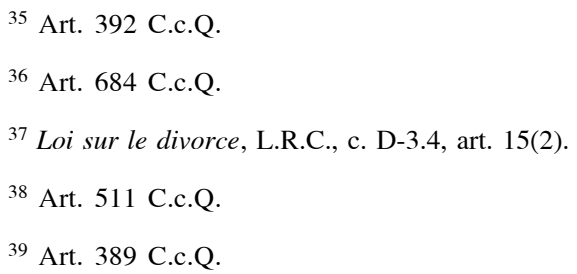

faire le législateur québécois a donc donné à l'union civile exactement les mêmes effets que ceux du mariage. ${ }^{40}$ Seul diffère le nom donné à l'union ainsi qu'à de l'acte de l'état civil qui la consigne. ${ }^{41}$ Cette seule différence suffit par ailleurs pour que la communauté homosexuelle persiste dans sa revendicaton du droit au mariage, la symbole du vocable demeurant à leurs yeux essentiel.

L'union de fait. Si l'union de droit est strictement réglementé afin d'imposer une conception associationniste au couple, le législateur québécois refuse, en principe, d'intervenir en matière d'union de fait afin de laisser aux concubins la liberté d'établir leur propre réglementation. Il s'agit là du dernier bastion de l'autonomie individuelle en matière de relation conjugale et celui-ci semble très important aux yeux de la societé québécoise. Conséquentement seules quelques rares dispositions du Code civil touchent l'union de fait, tels la possibilité pour le conjoint de fait de consentir aux soins de son partenaire devenu incapable de le faire $^{42}$ ou encore, en matière de bail résidentiel, le droit au maintien dans les lieux. ${ }^{43}$ Le régime primaire du mariage, que nous avons vu ainsi que l'obligation alimentaire, ne s'appliquent pas à l'union de

\footnotetext{
40 Art. 521.6 C.c.Q.

41 Art. 121.1 C.c.Q.

${ }^{42}$ Art. 15 C.c.Q.

43 Art. 1938 C.c.Q. Voir également l'article 1958
} C.c.Q. en matière de coproprieté indivis et les articles 555 et 579 C.c.Q, en matière d'adoption. 
fait. Les conjoints de fait qui le désirent doivent donc établir leurs droits réciproques par le biais d'une convention de vie commune dont la validité et l'exécution sont soumis au droit commun des obligations. Devant cet état des choses les tribunaux sont intervenus afin de permettre une certaine compensation économique au conjont de fait injustement défavorisé par la rupture de l'union. Ils le font, très exceptionnellement, par le biais de la reconnaissance d'une societé tacite lorsque les critères de celle-ci, dont celui de l'animus societatis, sont rencontrés ${ }^{44}$ et, plus fréquentement, par le biais de l'action en enrichissement injustifié. ${ }^{45}$

Parallèlement à cela les législateurs québécois et canadien ont étendu la notin de conjoint dans les différents lois fiscales et sociales afin d'y inclure les conjoints de fait hétérosexuels et homosexuels. Ces modifications, généralisées à la fin des années $90,{ }^{46}$ furent rendu nécessaires suite à certaines décisions jurisprudentielles qui avaient jugé discriminatoires, aux termes des chartes, le fait de limiter les avantages reconnus par ces lois aux seul couples mariés. ${ }^{47}$

44 Voir Dominique GOUBAU et Mireille D. CASTELLI, Précis de droit de la famille, Québec, PUL, 2001, p. 414.

45 Art. 1493 C.c.Q.

${ }^{46}$ Au Québec: Loi modifient diverses dispositions législatives concernant les conjoints de fait, L.Q. 1999, c. 14. Au fédéral: Loi sur la modernisation de certains régimes d'avantages et d'obligations, L.C. 2000 , c. 12.

47 Voir entre autres: Limieux c. Théorêt, [1999] R.J.Q. 1706 (C.S.); Miron c. Trudel, [1995] 2 R.C.S. 418. En ce qui concerne la discrimination et les couples de même sexe: Egan c. Canada, [1995] 2 R.C.S. 513; M. c. H. [1999] 2 R.C.S. 3.

\section{Dissolution de l'union}

Le mariage. Le mariage se dissout par le décès de l'un des conjoints ou par le divorce. ${ }^{48}$ Se rajoutent à ces motifs, la séparation de corps, moins fréquente, qui tout en laissant subsister le mariage délie les époux de l'obligation de faire vie commune ${ }^{49}$ et la nulitté qui anéantit l'union. ${ }^{50}$ Le divorce, quant à lui, étant de compétence fédérale, est régi par une loi unique pour l'ensemble du Canada qui en prévoit les conditions et les effets. La première loi sur le divorce fut adoptée en 1968. Avant cela, un couple québécois désirant divorcer devait obtenir une loi dite privée au Parlement fédéral.

La loi de 1968 exigeait pour l'obtention d'un divorce la preuve d'une faute de l'un des deux conjoints. ${ }^{51}$ Dans le but de rendre le processus moins pénible une seconde loi fut adoptée en 1985 qui établit que le seul motif de divorce est l'échec du mariage. Cet échec du mariage se prouve soit par l'adultère ou la cruauté physique ou mentale soit, beaucoup plus fréquemment, par la cessation de vie commune - voulue par au moins l'un des conjoints - qui, au jour du prononcé du divorce, aura duré au moins une année. ${ }^{52}$

La loi sur le divorce prévoit également la possibilité pour un époux de demander un support alimentaire sous forme de somme forfaitaire ou, plus généralement, de pension

\footnotetext{
48 Art. 516 C.c.Q.

49 Art. 507 C.c.Q.

${ }^{50}$ Art. 380 C.c.Q.

${ }^{51}$ Loi sur le divorce, L.C. 1967-1968, ch. 24,
} art. 3 .

${ }^{52}$ Loi sur le divorce, L.R.C., c. D-3.4, art. 8. 
à durée déterminée ou non. ${ }^{53}$ La Cour suprême a opté, dans les anées 1990, pour une conception plus associationiste du mariage et de ses effets post-rupture. Ainsi, l'obligation alimentaire peut avoir comme but tant l'indemnisation du préjudice économique subi par une partie conséquemment aux choix de vie de couple pendant l'union (par example le retrait de la vie active d'un des époux), ${ }^{54}$ mais aussi peut prendre sa base sur une «obligation sociale fondamentale» exigent du débiteur de voir à ce que les attentes de l'autre conjoint, crées par le modus vivendi de l'union, soient respectées même après l'union. ${ }^{55}$ Ce dernier fondement à l'obligation alimentaire, particulièrement flou, est diversement compris par les tribunaux d'instance inférieure, certains y voyant là un support alimentaire viager.

La garde des enfants ainsi que l'octroi d'aliments pour ceux-ci sont également prévus par la loi sur le divorce comme des accessoires au jugement en divorce lui-même. ${ }^{56}$

La dissolution du lien matrimonial (tout comme la nullité du mariage ou la séparation de corps) déclenche le partage du patrimoine familial, celui du régime matrimonial ainsi que, potentiellement, l'octroi d'une prestation compensatoire. Enfin, le tribunal peut attribuer le bail de la résidence familial au conjoint non locataire. ${ }^{57}$ Il peut également

\footnotetext{
${ }^{53}$ Id. Art. 15(2).

${ }^{54}$ Moge c.Moge, [1992] 1R.C.S. 183.

55 Bracklow c. Bracklow, [1999] 1 R.C.S. 420.

56 Id. Art. 15(1) et 16.

57 Art. 509 C.c.Q.
}

attribuer la propriété ou l'usage des meubles sevant à l'usage du ménage à l'époux non propriétaire ainsi que l'usage de la résidence familiale au conjoint à quil accorde la garde d'un nfant. ${ }^{58}$

L'union civile. Le divorce étant de compétence fédérale, le legislateur québécois ne pouvait pas automatiquement étendre celui-ci à l'union civile. ${ }^{59}$ Il a donc crée un régime de dissolution propre à l'union civile où il est possible pour les parties de mettre fin à l'union par une déclaration commune notariée, cette déclaration étant ensuite envoyée au Directeur de l'État civil. ${ }^{60}$ Cette rupture non judiciaire de l'union, non existante en matière de divorce, ne peut par ailleurs pas s'appliquer lorsque l'intérêt d'un enfant commun est en jeu. ${ }^{61}$ Dans ce cas, comme dans celui où les conjoints ne s'entendent pas, c'est le tribunal qui prononce la dissolution de l'union lorsque la volonté de vie commune des conjoints est irrémédiablement atteinte.

\section{LA FAMILLE PAR LA FILIATION}

\section{A. L'établissement du lien de filiation}

Trois modes d'établissement de a filiation coexistent dans le Code civil du Quebéc, le

58 Art. 410 C.c.Q.

59 Outre dissolution voluntaire ou judiciaire, l'union civile prend fin par le décès d'un des conjoints (art. 521.12 C.c.Q.). Quant à la nulitté de l'union civile pour vice de formation, elle enprunte les règles - dérogatoires au droit comun - de la nullité de mariage: art. 521.10 et 521.11 C.c.Q.
${ }^{60}$ Art. 521.13 C.c.Q.
61 Art. 521.17 C.c.Q. 
premier portant sur la filiation par le sang. Dans ce cas c'est l'acte de naissance qui est la preuve par ecellence du lien filial. Celuici est dressé par le Directeur de l'État civil à la suite de la déclaration par les parents de la naissance de l'enfant, et ce dans les trente jours de la naissance. ${ }^{62}$ Le lien filial peut subsidiarement être établi par une possession d'état constante ${ }^{63}$ ou par une reconnaissance volontaire. ${ }^{64}$ Enfin il existe une présomption réfragable selon laquelle le mari est le père de l'enfant de son épouse lorsque celui-ci naît pendant le mariage ou dans les trois cents jours de la dissolution. ${ }^{65}$ Depuis l'été 2002 cette présomption de paternité, qui prend sa source dans l'obligation de fidélité que se doivent les conjoints, s'applique également à l'union civile.

La filiation résultant d'un titre et d'une possession d'état conforme ne peut être contestée. ${ }^{66}$ Dans les autres cas il peut être nécessaire de recourir à un établissement judiciaire de la filiation. Dans un tel contexte il est maintenant possible pour le tribunal, sous certaines conditions, d'ordenner à une personne visée par l'action de subir une analyse par prélèvement d'une substance corporelle. ${ }^{67}$ Le tribunal peut tirer ensuite un présonption négative de tour refus injustifié.

Le lien filial peut également être établi par adoption. Celle-ci est prononcée par le

\footnotetext{
${ }^{62}$ Art. $113-114$ et 523 C.c.Q.

${ }^{63}$ Art. 524 C.c.Q.

${ }^{64}$ Art. 526 C.c.Q.

65 Art. 525 C.c.Q.

${ }^{66}$ Art. 530 C.c.Q.

67 Art. 535.1 C.c.Q.
}

tribunal compétent dans l'intérêt lorsque les parents y consentent ou encore lorsque cet enfant a été déclaré judiciairement admissible à l'adoption pour un motif prévu dans la loi. ${ }^{68}$ Selon l'article 546 C.c.Q. toute personne majeure, seulle ou conjointement avec une autre, peut adopter un enfant. Si cette disposition ne l'inclut pas expressément, il ne fait plus de doute, depuis les débats sur l'union civile et l'adoption de l'article 115 C.c.Q. qui précise la possibilité qu'un enfant ait des parents du même sexe,${ }^{69}$ que l'adoption par un couple de même sexe est permise en droit québécois. Le jugement en adoption confère à l'adopté une filiation qui se substitue à celle d'origine et qui fait naître les mêmes droits et obligations. ${ }^{70}$ La confidentialité des informations nominatives est garantie mais une procédure de «retrouvailles» existe lorsque l'enfant et les parents biologiques y consentent. ${ }^{71}$

Finalement le Code civil contient certaines dispositions concernant la procréacion assistée. Ces dispositions ont subi des modifications importantes dans le contexte de la loi sur l'union civile afin de les adapter à la realité «homoparentale». Ces dispositions s'appliquent á tout projet parental pour lequel une femme, seule ou avec son conjoint ou sa conjoint, a recours «aux forces genétique d'une personne qui n'est pas partie

\footnotetext{
68 Art. 559 C.c.Q.
}

${ }^{69}$ Cette disposition porte sur la déclaration de naissance. Lafin du premier alinéa se lit ainsi: «Lorsque les parents sont de même sexe, ils sont désignés comme les mères ou les pères de l'enfant selon le cas».
70 Art. 577-578 C.c.Q.
${ }^{71}$ Art. 582 C.c.Q. 
au projet parental». ${ }^{72}$ L'établissement de la filiation de l'enfant suit alors les mêmes règles que la filiation par le sang. ${ }^{73}$ Le titre est alors le moyen prioritaire afin d'établir la filiation. Le conjoint ou la conjointe qui a consenti au projet parental peut donc, tout comme la mère, déclarer l'enfant à sa naissance. ${ }^{74}$ Mais surtout le conjoint marié ou uni civilement - incluant dans le dernier cas la conjoint de même sexe - sera présumé être le père ou la co-mère de l'enfant si celuici naît pendant l'union ou à l'intérieur de trois cents jours après la dissolution. ${ }^{75}$ Cette présomption de «parenté» s'appliquant au couple de même sexe est certainement l'introduction la plus révolutionnaire et la plus contestée, malgré l'absence de débat et la rapidité étonnante de son adoption, du droit québécois. À notre connaissance elle est unique.

Le recours à un tiers dans le cadre du projet parental peut certes se faire le biais des techniques médicales mais peut prendre forme dans une relation sexuelle où l'homme agit uniquement comme "géniteur». ${ }^{76} \mathrm{Si}$ dans le premier cas le tiers donneur ne peut revendiquer aucun lien de filiation avec l'enfant [d'ailleurs l'identité du donneur est conservée confidentielle], dans le second l'homme peut, s'il le désire, revindiquer un lien de filiation dans l'année suivant la naissance de l'enfant. ${ }^{77}$ Cette conception de

\footnotetext{
72 Art. 538 C.c.Q.

73 Art. 538.1 C.c.Q.

74 Art. 115 C.c.Q.

75 Art. 538.3 C.c.Q.

76 Art. 538.2 C.c.Q.

77 Art. 538.a al. 2 C.c.Q.
}

l'assistance à la procréation, qui date également de la réforme sur l'union civile, même si elle est sujette à s'appliquer à d'autres situations, vise celle des couples de femmes qui, plutôt que de s'adresser à une clinique de fertilité, demande à un ami de colaborer à leur projet parental.

\section{B. Les effets de la filiation}

Les différences qui exitaient dans l'ancien code entre les enfants légitimes, naturels, adultérins et incestueux sont disparues depuis la réforme de 1980 qui a établi péremptoirement l'égalité de tous les enfants quelles que soint les circonstances de leur naissance. ${ }^{78}$ Tout enfant possède donc les mêmes droits et devoirs envers ses parents; il hérite $a b$ intestat, ${ }^{79}$ est créancier d'aliments ${ }^{80}$ et leur doit, à tout âge, respect. ${ }^{81}$

L'enfant reste sous l'autorité de ses parents, jusqu'à la majorité ou son émancipation. ${ }^{82}$ Depuis une réforme survenue en $1977,{ }^{83}$ l'authorité parentale remplace la puissance paternelle. Les parents exercent ensemble cette autorité parentale, ${ }^{84}$ ce qui signifie qu'ils ont à l'égard de leur enfant le droit et devoir de garde de surveillence et l'éducation et doivent le nourrir et l'entretenir. ${ }^{85}$ Ils décident ensemble du nom, le nom de famille puvant

\footnotetext{
78 Art. 522 C.c.Q.

${ }^{79}$ Art. 666 et suiv. C.c.Q.

${ }^{80}$ Art. 585 C.c.Q.

81 Art. 597 C.c.Q.

82 Art. 598 C.c.Q.

${ }^{83}$ Loi modifient le Code civil, L.Q. 1977, c. 72.

${ }^{84}$ Art. 600 C.c.Q.

85 Art. 599 C.c.Q.
} 
être celui de la mère, du père ou des deux. ${ }^{86}$ Les parents sont également tuteurs légaux de leur enfant. ${ }^{87}$ À ce titre ils administrent son patrimoine et exercent ses droits civils. En cas de conflit dans l'exercice de l'autorité parentale le tribunal doit statuer en fonction de l'intérêt de l'enfant. ${ }^{88}$

\section{L'obligation alimentaire}

L'obligation alimentaire issue du lien filial est réduite à sa plus simple expression, l'article 585 C.c.Q la limitant aux seuls parents en ligne directe au premier degré. Jusqu'en 1996 cette obligation existait en ligne directe au-delà du premier degré mais l'obligation alimentaire des grands-parents fut, suite à des revendications des personnes âgées, abrogée.

Cette obligation alimentaire n'est aucunement limitée dans les temps. Les tribunaux reconnaissent don le droit aux enfants majeurs qui ne peuvent subvenir seuls à leur besoin, par exemple parce qu'ils sont encore aux études, de demander des aliments à leur parents. ${ }^{89}$ Évidemment l'attribution des aliments étant toujours centré autour des besoins et des facultrés des parties, ${ }^{90}$ les tribunaux exigent de l'enfant majeur une participation personnelle à sa subsistance.

Tout comme pour l'obligation alimentaire entre les ex-conjoints, celle issue du lien filial subsiste, mais de façon éphémère au

\footnotetext{
${ }^{86}$ Art. 51 C.c.Q.

${ }^{87}$ Art. 192 C.c.Q.

88 Art. 604 C.c.Q.
}

${ }^{89}$ D. GOUBAU et M. D. CASTELLI, op. cit., note 44, p. 279; Monique OULLETTE, Droit de la famille, 3e éd., Montréal, Thémis, 1995, p. 287.

90 Art. 587 C.c.Q. décès du débiteur. L'enfant, par example, peut donc demander à la sucession, dans les six mois du décès, un support alimentaire. ${ }^{91}$ Celui-ci est octroyé en fonctions de diférents critères dont, certes, les besoins et facultés du créancier mais également des avantages qu'il a reçus dans le cadre de la succesion. ${ }^{92}$ Cette survie de l'obligation alimentaire ne constitue donc pas une réserve héréditaire, étrangère au principe de la liberté de tester prévalant en droit québécois.

\section{CONCLUSION}

Ce bref aperçu du droit québécois de la famille laisse que les multiples réformes en cette matière ont toutes convergé, dans les trent dernière années, vers l'établissement d'une égalité entre les membres constituant de la famille. Égalité juridique en un premier temps, tant entre les conjoints que les enfants, mais également égalité économique de ceuxci. Si le droit de la famille avait jadis plus pour but de protéger l'institution du mariage en évitant l'éclatement de celui-ci, allant jusqu'à le «proscrire», il s'attarde maintenant plutôt vers la protection des membres de la famille tout particulièrement dans l'éventualité d'un rupture de la cellule familiae. Le prochain défi du droit québécois de la famille semble maintenant devoir être la prise en compte de l'éclatement du modèle familial unique au profit d'une réalité plurale. Sur ce point le droit québécois vient de s'adapter aux familles homosexuelles, mais il lui rest encore bien des défis à relever, tel celui de la famille recomposée qui est très largement ignorée de nos législations.

\footnotetext{
91 Art. 684 C.c.Q.

${ }^{92}$ Art. 686 C.c.Q.
} 
